WiKTor OkTaba and JoAnna TARAsińska (Lublin)

\title{
ESTIMATION OF THE GENERALIZED VARIANCE IN A BIVARIATE NORMAL DISTRIBUTION FROM AN INCOMPLETE SAMPLE
}

Abstract. The aim of the paper is estimation of the generalized variance of a bivariate normal distribution in the case of a sample with missing observations. The estimator based on all available observations is compared with the estimator based only on complete pairs of observations.

1. Introduction. Let a random variable $(y, z)$ have normal distribution with mean $\boldsymbol{\mu}=\left[\mu_{1}, \mu_{2}\right]^{\prime}$ and variance-covariance matrix $\boldsymbol{\Sigma}=\left[\begin{array}{cc}\sigma_{y}^{2} & \sigma_{y z} \\ \sigma_{y z} & \sigma_{z}^{2}\end{array}\right]$ :

$$
(y, z) \sim N_{2}\left(\left[\begin{array}{l}
\mu_{1} \\
\mu_{2}
\end{array}\right], \boldsymbol{\Sigma}\right) .
$$

Let $[\mathbf{y}, \mathbf{z}]$ be a simple random sample of size $k$ from the distribution (1). We are interested in estimation of the generalized variance, i.e. the determinant $|\boldsymbol{\Sigma}|$. The generalized variance is used in various statistical analyses concerning the covariance structure of the model.

The sample generalized variance

$$
|\mathbf{S}|=\left|\begin{array}{ll}
\frac{1}{k-1} \sum_{i=1}^{k}\left(y_{i}-\bar{y}\right)^{2} & \frac{1}{k-1} \sum_{i=1}^{k}\left(y_{i}-\bar{y}\right)\left(z_{i}-\bar{z}\right) \\
\frac{1}{k-1} \sum_{i=1}^{k}\left(y_{i}-\bar{y}\right)\left(z_{i}-\bar{z}\right) & \frac{1}{k-1} \sum_{i=1}^{k}\left(z_{i}-\bar{z}\right)^{2}
\end{array}\right|,
$$

where $\bar{y}=k^{-1} \sum_{i=1}^{k} y_{i}, \bar{z}=k^{-1} \sum_{i=1}^{k} z_{i}$, is very well investigated ([1], [7],

2000 Mathematics Subject Classification: 62F10, 62H12.

Key words and phrases: bivariate normal distribution, missing data, generalized variance, complete-case estimator, available-case estimator. 
[5], [3], [4]). It is known for example that

$$
\frac{|(k-1) \mathbf{S}|}{|\boldsymbol{\Sigma}|}=\chi_{k-1}^{2} \cdot \chi_{k-2}^{2},
$$

where $\chi_{k-1}^{2}$ and $\chi_{k-2}^{2}$ are independently $\chi^{2}$ distributed with $k-1$ and $k-2$ degrees of freedom, respectively. Thus

$$
\frac{k-1}{k-2}|\mathbf{S}|=\frac{1}{(k-1)(k-2)}\left|\begin{array}{ll}
\sum_{i=1}^{k}\left(y_{i}-\bar{y}\right)^{2} & \sum_{i=1}^{k}\left(y_{i}-\bar{y}\right)\left(z_{i}-\bar{z}\right) \\
\sum_{i=1}^{k}\left(y_{i}-\bar{y}\right)\left(z_{i}-\bar{z}\right) & \sum_{i=1}^{k}\left(z_{i}-\bar{z}\right)^{2}
\end{array}\right|
$$

is an unbiased estimator of $|\boldsymbol{\Sigma}|$ and

$$
\operatorname{Var}\left(\frac{k-1}{k-2}|\mathbf{S}|\right)=\frac{2|\boldsymbol{\Sigma}|^{2}(2 k-1)}{(k-1)(k-2)} .
$$

2. Estimation of $|\Sigma|$ in the case of missing observations. Let us consider an incomplete sample

$$
\left[\begin{array}{ccccccccc}
y_{1} & \ldots & y_{k} & y_{k+1} & \ldots & y_{k+p} & * & \ldots & * \\
z_{1} & \ldots & z_{k} & * & \ldots & * & z_{k+p+1} & \ldots & z_{k+p+s}
\end{array}\right]^{\prime},
$$

where $*$ denotes an observation missing completely at random ([2], [6]). So, we have $k$ complete pairs of observations, $p$ additional observations of the $y$ variable and $s$ additional observations of the $z$ variable. To simplify let us write the sample in the following form:

\begin{tabular}{|c|c|}
\hline $\mathbf{y}_{0}$ & $\mathbf{z}_{0}$ \\
\hline $\mathbf{y}_{1}$ & $*$ \\
\hline$*$ & $\mathbf{z}_{2}$ \\
\hline
\end{tabular}

where $\mathbf{y}_{0}=\left[\mathbf{y}_{1}, \ldots, \mathbf{y}_{k}\right]^{\prime}, \mathbf{z}_{0}=\left[\mathbf{z}_{1}, \ldots, \mathbf{z}_{k}\right]^{\prime}, \mathbf{y}_{1}=\left[\mathbf{y}_{k+1}, \ldots, \mathbf{y}_{k+p}\right]^{\prime}, \mathbf{z}_{2}=$ $\left[\mathbf{z}_{k+p+1}, \ldots, \mathbf{z}_{k+p+s}\right]^{\prime}$. Let us set

$$
\mathbf{y}=\left[\begin{array}{l}
\mathbf{y}_{0} \\
\mathbf{y}_{1}
\end{array}\right], \quad \mathbf{z}=\left[\begin{array}{l}
\mathbf{z}_{0} \\
\mathbf{z}_{2}
\end{array}\right] .
$$

The question is: how should we estimate $|\boldsymbol{\Sigma}|$ using the additional information contained in the vectors $\mathbf{y}_{1}$ and $\mathbf{z}_{2}$ and is it worth doing? Perhaps the estimator based on complete pairs $\left[\mathbf{y}_{0}, \mathbf{z}_{0}\right]$ (complete-case estimator) is better?

As an alternative to the complete-case estimator we consider the available-case estimator which uses all the available values to estimate parame- 
ters in model (1). To estimate $|\boldsymbol{\Sigma}|$ we use the following sums:

$$
\sum_{i=1}^{k+p}\left(y_{i}-\bar{y}\right)^{2}, \quad \sum_{i=1}^{k}\left(z_{i}-\bar{z}\right)^{2}+\sum_{i=k+p+1}^{k+p+s}\left(z_{i}-\bar{z}\right)^{2}, \quad \sum_{i=1}^{k}\left(y_{i}-\bar{y}\right)\left(z_{i}-\bar{z}\right)
$$

where $\bar{y}$ and $\bar{z}$ are the arithmetic means of elements of $\mathbf{y}$ and $\mathbf{z}$, respectively. Each of these sums, multiplied by a suitable constant, is a better unbiased estimator of $\sigma_{y}^{2}, \sigma_{z}^{2}, \sigma_{y z}$ than the complete-case estimators

$$
\frac{1}{k-1} \sum_{i=1}^{k}\left(y_{i}-\bar{y}_{0}\right)^{2}, \quad \frac{1}{k-1} \sum_{i=1}^{k}\left(z_{i}-\bar{z}_{0}\right)^{2}, \quad \frac{1}{k-1} \sum_{i=1}^{k}\left(y_{i}-\bar{y}_{0}\right)\left(z_{i}-\bar{z}_{0}\right),
$$

where $\bar{y}_{0}$ and $\bar{z}_{0}$ are the means of $\mathbf{y}_{0}$ and $\mathbf{z}_{0}$.

Let us consider the following estimate of $|\boldsymbol{\Sigma}|$ :

$$
\begin{aligned}
E= & a \cdot \sum_{i=1}^{k+p}\left(y_{i}-\bar{y}\right)^{2} \cdot\left[\sum_{i=1}^{k}\left(z_{i}-\bar{z}\right)^{2}+\sum_{i=k+p+1}^{k+p+s}\left(z_{i}-\bar{z}\right)^{2}\right] \\
& -b \cdot\left(\sum_{i=1}^{k}\left(y_{i}-\bar{y}\right)\left(z_{i}-\bar{z}\right)\right)^{2}
\end{aligned}
$$

where $a$ and $b$ are constants (depending on $k, p, s$ ) giving unbiasedness of $E$. To determine $a$ and $b$ and then to calculate the variance of $E$ we use the results of Wilks [8]. He considered the following random variables for the incomplete sample (5):

$$
\begin{gathered}
\xi_{0}=\frac{1}{k+p} \sum_{i=1}^{k+p}\left(y_{i}-\bar{y}\right)^{2}, \quad \eta_{0}=\frac{1}{k+s}\left(\sum_{i=1}^{k}\left(z_{i}-\bar{z}\right)^{2}+\sum_{i=k+p+1}^{k+p+s}\left(z_{i}-\bar{z}\right)^{2}\right), \\
\zeta_{0}=\frac{1}{k} \sum_{i=1}^{k}\left(y_{i}-\bar{y}\right)\left(z_{i}-\bar{z}\right),
\end{gathered}
$$

and found the moment generating function

$$
\varphi(\gamma, \delta, \varepsilon)=E\left(e^{\gamma \xi_{0}+\delta \eta_{0}+\varepsilon \zeta_{0}}\right)
$$

which can be used for finding joint moments of $\left(\xi_{0}, \eta_{0}, \zeta_{0}\right)$ :

$$
E\left(\xi_{0}^{h} \eta_{0}^{k} \zeta_{0}^{l}\right)=M(h, k, l)=\left.\frac{\partial^{h} \partial^{k} \partial^{l}}{\partial \gamma^{h} \partial \delta^{k} \partial \varepsilon^{l}} \varphi(\gamma, \delta, \varepsilon)\right|_{\gamma=\delta=\varepsilon=0} .
$$

We have used $\varphi(\gamma, \delta, \varepsilon)$ to obtain the required moments of sums (6). All 
computations were done by using Maple $\mathrm{V}$. The values of $a$ and $b$ are

$$
\begin{aligned}
a & =\frac{2(k-1)+c+c^{2}+(k-1+c)^{2}}{(k+p-1)(k+s-1)\left[k-1+c^{2}+(k-1+c)^{2}\right]-2(k-1+c)^{2}}, \\
b & =\frac{(k+p-1)(k+s-1)+2(k-1+c)}{(k+p-1)(k+s-1)\left[k-1+c^{2}+(k-1+c)^{2}\right]-2(k-1+c)^{2}},
\end{aligned}
$$

where $c=\frac{p s}{(k+p)(k+s)}$. When $s=0, a$ and $b$ have a simpler form:

$$
a=\frac{k+1}{(k-1)\left(k^{2}-k+p k-2\right)}, \quad b=\frac{k+p+1}{(k-1)\left(k^{2}-k+p k-2\right)} .
$$

For a complete sample $(p=s=0)$ we have the known values

$$
a=b=\frac{1}{(k-1)(k-2)}
$$

(see (3)). The variance of $E$ is

$$
\begin{aligned}
\operatorname{Var}(E)= & a^{2}(k+p)^{2}(k+s)^{2}\left[M(2,2,0)-M(1,1,0)^{2}\right] \\
& +b^{2} k^{4}\left[M(0,0,4)-M(0,0,2)^{2}\right] \\
& -2 a b k^{2}(k+p)(k+s)[M(1,1,2)-M(1,1,0) \cdot M(0,0,2)] .
\end{aligned}
$$

We do not give here the expressions for the moments $M(h, k, l)$ because they are long and complicated (especially $M(2,2,0), M(0,0,4), M(1,1,2))$. We are interested in comparing the estimator $E$ given by (7) and the estimator $E_{0}$ based on complete pairs of observations:

$$
E_{0}=\frac{1}{(k-1)(k-2)}\left[\sum_{i=1}^{k}\left(y_{i}-\bar{y}_{0}\right)^{2} \cdot \sum_{i=1}^{k}\left(z_{i}-\bar{z}_{0}\right)^{2}-\left(\sum_{i=1}^{k}\left(y_{i}-\bar{y}_{0}\right)\left(z_{i}-\bar{z}_{0}\right)\right)^{2}\right] .
$$

When $s=0$ we get a simple equation

$$
\operatorname{Var}(E)-\operatorname{Var}\left(E_{0}\right)=\frac{-2 p \sigma_{y}^{4} \sigma_{z}^{4}(k+1)\left[A \varrho^{4}+B \varrho^{2}+C\right]}{(k-2)(k-1)\left(k^{2}+p k-k-2\right)^{2}},
$$

where $A=4(k+1)(k-2)+2 p k, B=-2\left(k^{2}-4\right)(k+p+1)-4 p k, C=$ $(k-2)\left(k^{2}-1\right)+p\left(k^{2}-k+2\right)$ and $\varrho$ is the correlation coefficient between $y$ and $z$.

Superiority of one estimator over the other depends on $\varrho^{2}, k, p$, namely $E$ is better when $\varrho^{2}<f(k, p)$ and $E_{0}$ is better when $\varrho^{2}>f(k, p)$, where $f(k, p)$ is the smaller root of the quadratic equation $A x^{2}+B x+C=0$. Analysing $f(k, p)$ we can state the following simple corollary:

Corollary 1. If $\varrho^{2} \leq 0.3$ than $E$ is better than $E_{0}$ for each $k>3$ and for each $p>0$. If $\varrho^{2} \geq 0.5$ then $E_{0}$ is better than $E$ for each $k \geq 3$ and for each $p>0$. 
The case $s=0$ can be applied to the situation when getting an observation of one variable (for example $z$ ) is much more difficult or expensive than for the other $(y)$. Suppose we have $k$ complete pairs of observations. The question is: how large is $p_{0}$, the number of additional observations of $y$ that cause at least the same decrease of variance of $E$ as one additional complete pair? Using Maple $\mathrm{V}$ we get the following answer:

Corollary 2. - If $|\varrho| \leq 0.3$ and $k \geq 10$ then $p_{0}=3$.

- If $|\varrho| \leq 0.5$ and $k \geq 10$ then $p_{0}=5$.

- If $|\varrho| \leq 0.5$ and $k \geq 20$ then $p_{0}=3$.

When $s>0$ then the difference $\operatorname{Var}(E)-\operatorname{Var}\left(E_{0}\right)$ is not so simple as in (8) and we do not give here the long expression for that. Let us only state that $\operatorname{Var}(E)$ is symmetric in $p$ and $s$, that is,

$$
\operatorname{Var}(E)_{(k, p, s)}=\operatorname{Var}(E)_{(k, s, p)} .
$$

In Tables $1,2,3$ and 4 we give the values of $\operatorname{Var}(E) / \operatorname{Var}\left(E_{0}\right)$ for various $k, p, s$ and $\varrho$. The upper value in the tables is for $|\varrho|=0.3$, the middle one for $|\varrho|=0.5$ and the lower one for $|\varrho|=0.8$.

So the estimator $E$ can be either much better or much worse than $E_{0}$. $E$ is not recommended when $|\varrho|$ is greater than 0.5 . Unfortunately $E$ has one disadvantage: theoretically it can have a negative value. We tried to estimate how often it can happen using Maple V simulation. We generated 1000 samples from a bivariate normal distribution with $\mu_{1}=\mu_{2}=0, \sigma_{y}^{2}=\sigma_{z}^{2}=1$, $\varrho=0.5$ for different $k, p, s$. The results of this simulation in Table 5 show that the probability of getting negative values of $E$ is small.

Table 1. $k=10$

\begin{tabular}{|l|c|c|c|c|}
\hline \multicolumn{1}{|c|}{$p$} & 2 & 5 & 10 & 15 \\
$s$ & & & & \\
\hline \multirow{3}{*}{0} & 0.910 & 0.824 & 0.740 & 0.690 \\
& 0.937 & 0.875 & 0.814 & 0.778 \\
& 1.392 & 1.770 & 2.135 & 2.348 \\
\hline \multirow{3}{*}{2} & 0.827 & 0.745 & 0.666 & 0.620 \\
& 0.887 & 0.837 & 0.787 & 0.757 \\
& 1.898 & 2.377 & 2.830 & 3.091 \\
\hline \multirow{3}{*}{5} & & 0.669 & 0.595 & 0.552 \\
& & 0.798 & 0.759 & 0.735 \\
& & 2.948 & 3.488 & 3.798 \\
\hline \multirow{3}{*}{10} & & & 0.526 & 0.486 \\
& & & 0.731 & 0.713 \\
& & & 4.112 & 4.472 \\
\hline \multirow{2}{*}{15} & & & & 0.447 \\
& & & & 0.700 \\
& & & & 4.861 \\
\hline
\end{tabular}

Table 2. $k=20$

\begin{tabular}{|c|c|c|c|c|}
\hline \multicolumn{1}{|c|}{$p$} & 5 & 10 & 15 & 20 \\
$s$ & & & & \\
\hline \multirow{3}{*}{0} & 0.897 & 0.830 & 0.782 & 0.747 \\
& 0.917 & 0.863 & 0.825 & 0.796 \\
& 1.331 & 1.552 & 1.710 & 1.829 \\
\hline \multirow{3}{*}{5} & 0.800 & 0.738 & 0.693 & 0.660 \\
& 0.853 & 0.811 & 0.781 & 0.759 \\
& 1.852 & 2.193 & 2.434 & 2.613 \\
\hline \multirow{3}{*}{10} & & 0.677 & 0.634 & 0.602 \\
& & 0.777 & 0.752 & 0.734 \\
& & 2.612 & 2.906 & 3.125 \\
\hline \multirow{3}{*}{15} & & & 0.593 & 0.562 \\
& & & 0.732 & 0.717 \\
& & & 3.239 & 3.486 \\
\hline \multirow{2}{*}{20} & & & & 0.532 \\
& & & & 0.704 \\
& & & & 3.753 \\
\hline
\end{tabular}


Table 3. $k=50$

\begin{tabular}{|c|c|c|c|c|c|}
\hline${ }_{s} p$ & 10 & 20 & 30 & 40 & 50 \\
\hline \multirow{3}{*}{0} & 0.916 & 0.857 & 0.812 & 0.778 & 0.750 \\
\hline & 0.928 & 0.877 & 0.838 & 0.801 & 0.758 \\
\hline & 1.216 & 1.372 & 1.489 & 1.580 & 1.653 \\
\hline \multirow{3}{*}{10} & 0.836 & 0.779 & 0.737 & 0.704 & 0.677 \\
\hline & 0.868 & 0.826 & 0.795 & 0.770 & 0.751 \\
\hline & 1.570 & 1.821 & 2.010 & 2.156 & 2.273 \\
\hline \multirow{3}{*}{20} & & 0.724 & 0.683 & 0.651 & 0.625 \\
\hline & & 0.790 & 0.764 & 0.743 & 0.726 \\
\hline & & 2.141 & 2.380 & 2.565 & 2.713 \\
\hline \multirow{3}{*}{30} & & & 0.643 & 0.611 & 0.587 \\
\hline & & & 0.740 & 0.722 & 0.708 \\
\hline & & & 2.656 & 2.870 & 3.041 \\
\hline \multirow{3}{*}{40} & & & & 0.581 & 0.556 \\
\hline & & & & 0.706 & 0.694 \\
\hline & & & & 3.107 & 3.296 \\
\hline \multirow{3}{*}{50} & & & & & 0.532 \\
\hline & & & & & 0.682 \\
\hline & & & & & 3.499 \\
\hline
\end{tabular}

Table 4. $k=100$

\begin{tabular}{|c|c|c|c|c|c|}
\hline${ }_{s}^{p}$ & 20 & 40 & 60 & 80 & 100 \\
\hline \multirow{3}{*}{0} & 0.917 & 0.858 & 0.813 & 0.779 & 0.751 \\
\hline & 0.927 & 0.875 & 0.836 & 0.806 & 0.781 \\
\hline & 1.198 & 1.340 & 1.446 & 1.530 & 1.596 \\
\hline \multirow{3}{*}{20} & 0.837 & 0.780 & 0.738 & 0.705 & 0.678 \\
\hline & 0.866 & 0.823 & 0.791 & 0.766 & 0.746 \\
\hline & 1.533 & 1.772 & 1.951 & 2.091 & 2.202 \\
\hline \multirow{3}{*}{40} & & 0.725 & 0.684 & 0.652 & 0.626 \\
\hline & & 0.786 & 0.759 & 0.737 & 0.720 \\
\hline & & 2.080 & 2.311 & 2.491 & 2.634 \\
\hline \multirow{3}{*}{60} & & & 0.643 & 0.612 & 0.587 \\
\hline & & & 0.735 & 0.716 & 0.701 \\
\hline & & & 2.581 & 2.790 & 2.957 \\
\hline \multirow{3}{*}{80} & & & & 0.581 & 2.557 \\
\hline & & & & 0.700 & 0.687 \\
\hline & & & & 3.022 & 3.208 \\
\hline \multirow{3}{*}{100} & & & & & 0.532 \\
\hline & & & & & 0.675 \\
\hline & & & & & 3.408 \\
\hline
\end{tabular}

Table 5. The number of negative values of $E$ (per 1000 samples)

\begin{tabular}{|c|c|c|c|c|c|c|c|c|}
\hline \multicolumn{5}{|c|}{$k=10$} & \multicolumn{2}{|c|}{$k=20$} & $k=50$ & $k=100$ \\
\hline $\begin{array}{l}p=5 \\
s=0\end{array}$ & $\begin{array}{l}p=5 \\
s=5\end{array}$ & $\begin{array}{l}p=10 \\
s=0\end{array}$ & $\begin{array}{l}p=10 \\
s=5\end{array}$ & $\begin{array}{l}p=10 \\
s=10\end{array}$ & $\begin{array}{l}p=10 \\
s=10\end{array}$ & $\begin{array}{l}p=20 \\
s=20\end{array}$ & $\begin{array}{l}p=50 \\
s=50\end{array}$ & $\begin{array}{l}p=100 \\
s=100\end{array}$ \\
\hline 3 & 18 & 10 & 21 & 40 & 0 & 6 & 0 & 0 \\
\hline
\end{tabular}

\section{References}

[1] T. W. Anderson, An Introduction to Multivariate Statistical Analysis, Wiley, New York, 1958.

[2] R. J. A. Little and D. B. Rubin, Statistical Analysis with Missing Data, Wiley, New York, 1987.

[3] W. Oktaba, Densities of determinant ratios, their moments and some simultaneous confidence intervals in the multivariate Gauss-Markoff model, Appl. Math. 40 (1995), $47-54$.

[4] - Asymptotically normal confidence intervals for a determinant in a generalized multivariate Gauss-Markoff model, ibid., 55-59.

[5] C. R. Rao, Linear Statistical Inference and Its Applications, Wiley, New York, 1973.

[6] D. B. Rubin, Inference and missing data, Biometrika 63 (1976), 581-592.

[7] M. S. Srivastava and C. G. Khatri, An Introduction to Multivariate Statistics, NorthHolland, New York, 1979. 
[8] S. S. Wilks, Moments and distributions of estimates of population parameters from fragmentary samples, Ann. Math. Statist. 3 (1932), 163-195.

Department of Mathematical Statistics

Institute of Applied Mathematics

Academy of Agriculture

Akademicka 13

20-934 Lublin, Poland

E-mail: johata@ursus.ar.lublin.pl

Received on 28.10.1999;

revised version on 27.9.2000

(1508) 